\title{
A posteriori metadata from automated provenance tracking: integration of AiiDA and TCOD
}

\author{
Andrius Merkys ${ }^{1,2^{*}}$ (D) Nicolas Mounet ${ }^{1}$, Andrea Cepellotti ${ }^{1}$, Nicola Marzari', Saulius Gražulis 2,3 (i) \\ and Giovanni Pizzi ${ }^{1}$
}

\begin{abstract}
In order to make results of computational scientific research findable, accessible, interoperable and re-usable, it is necessary to decorate them with standardised metadata. However, there are a number of technical and practical challenges that make this process difficult to achieve in practice. Here the implementation of a protocol is presented to tag crystal structures with their computed properties, without the need of human intervention to curate the data. This protocol leverages the capabilities of AiiDA, an open-source platform to manage and automate scientific computational workflows, and the TCOD, an open-access database storing computed materials properties using a well-defined and exhaustive ontology. Based on these, the complete procedure to deposit computed data in the TCOD database is automated. All relevant metadata are extracted from the full provenance information that AiiDA tracks and stores automatically while managing the calculations. Such a protocol also enables reproducibility of scientific data in the field of computational materials science. As a proof of concept, the AiiDA-TCOD interface is used to deposit 170 theoretical structures together with their computed properties and their full provenance graphs, consisting in over 4600 AiiDA nodes.
\end{abstract}

Keywords: DFT, Reproducibility, Provenance, Open data, Ontology, Materials science

\section{Background}

Modelling and simulation are commonly identified as the third paradigm in scientific understanding, complementing theory and experiment. In particular, computational materials science has developed into an essential field due to two main factors. First, in the past years significant advances have been achieved both in the approximations of the theories used to simulate materials from first-principles [1] and in the codes that implement them (many of which are distributed open-source). Second, these computationally-expensive calculations have been made feasible thanks to the exponential increase of computing power predicted by Moore's law and the corresponding decrease of the price/performance ratio. As a

\footnotetext{
*Correspondence: andrius.merkys@gmail.com

${ }^{2}$ Institute of Biotechnology, Vilnius University, Saulètekio al. 7,

10257 Vilnius, Lithuania

Full list of author information is available at the end of the article
}

consequence, large number of properties can nowadays be computed for large families of materials. A number of online databases has appeared in the past few years, like the Materials Project [2], OQMD [3] and AFLOWLIB [4]. However, much effort is still needed to consolidate the knowledge from publications, tagging results with suitable metadata under an established ontology, and preserving at the same time the complete provenance of the computed data to enable reproducibility of the results.

Currently, there are several attempts to define an ontology in the field of theoretical material science, like the European Theoretical Spectroscopy Facility (ETSF) [5, 6], NOMAD [7], OPTiMaDe [8] and the Theoretical Crystallography Open Database (TCOD) [9, 10]. The latter was launched with the aim of collecting the results from several kinds of calculations (DFT, post-HF, $\mathrm{QM} / \mathrm{MM}$, etc.), into an open-access resource for longterm archival storage. The TCOD adopts the Crystallographic Information Framework (CIF) format [11], 
a unified format for reporting and storing the results of experimentally-solved crystal structures, which has been widely adopted and used as the de facto standard by most crystallographic journals as well as structural databases like, to mention just a few, the Inorganic Crystal Structure Database (ICSD) [12], the Cambridge Structural Database [13], the American Mineralogist Crystal Structure Database [14], and the Crystallography Open Database (COD) $[15,16]$. One of the main advantages of the CIF format is the existence of CIF dictionaries, aimed at defining domain-specific ontologies readable both by humans and by machines [17]. Constraints, units of measurement and interrelationships for data values are specified in order to homogenise the data, eliminate ambiguities and allow for automated validation. Furthermore, since CIF data names are uniquely defined, a CIF file may contain properties from more than one dictionary, making it possible to easily extend and complement the file (e.g. for macromolecular crystallography [18], powder diffraction [19], electron density [20] and experimental material properties [21]). Recently, CIF format version 2.0 (CIF 2, [22]) has been developed with even more features for ontology definition. In the TCOD domain-specific dictionaries have been compiled in order to define an ontology for the hosted data (in particular, the cif_dft.dic dictionary for DFT-based properties, and cif_tcod.dic for the generic metadata related to scientific workflows), and automated checks of CIF files against these dictionaries have been implemented.

The definition of an ontology is not the only challenge that materials science faces; another major issue is the preservation of provenance for result replication. In fact, currently most of the scientific publications provide only a subset of all control parameters, numerical inputs and calculation interrelationships needed to exactly reproduce the published results. This problem can be solved by using provenance-tracking frameworks like AiiDA [23, 24], a high-throughput infrastructure that provides a high-level research environment to automate the execution of computations, systematically store inputs and outputs and their relationships in a graph database (tailored to keep track of the full data provenance) and share results.

In this work we present the integration of the TCOD database with AiiDA, using and enhancing the cif tcod. dic CIF dictionary [25]. Our integration of a calculation automation framework and an ontology-based database allows for the a posteriori deposition of simulation results with automatically recorded metadata. Most importantly, the metadata tagging and deposition can be performed at any time after the calculation has been executed thanks to the automatic provenance tracking provided by AiiDA.
In the following, we first describe the provenance model implemented in AiiDA and explain how we map it to a CIF file. We explain how we address and solve the technical issues that arise in the process, e.g. the inclusion of input and output files within the CIF file and their encoding, how software versions can be tracked, and how to report bibliographic references. We then describe the extensions to the TCOD dictionary that we have implemented, and compare the latter with other existing ontologies. The algorithm of the converters to integrate AiiDA and the TCOD is then illustrated. Finally, we discuss the results obtained and deposited in the TCOD using the codes and algorithms implemented in this work.

\section{Workflow representation}

\section{Provenance model and directed acyclic graphs}

Reproducibility of scientific calculations is a crucial tenet of computational scientific research [26-28], and a system enabling easy replication of exact or modified computations reported in a scientific publication would be an important step in achieving this goal [29]. An important prerequisite is that content (data) must be separated from its presentation (article) [30]. Such separation is implemented for instance in Sweave [31], designed specifically for the $R$ statistical programming language [32]. In the field of Computational Materials Science, Pizzi et al. have developed AiiDA, a Python-based framework for atomistic simulations [24], where data provenance is stored automatically while the simulations and workflows are executed, using a data model elaborating on ideas of the Open Provenance Model (OPM) [33, 34]. OPM suggests to represent whole scientific workflows of data transformations as directed acyclic graphs (DAGs), whose nodes are artifacts and vertices are processes. An example of DAG of an AiiDA workflow is shown in Fig. 1.

The purpose of this work is to represent faithfully the full provenance of computational workflows, represented by AiiDA in the form of DAGs, within a CIF file. To achieve this goal, we apply the cif_tcod.dic CIF dictionary as follows. We represent workflows by an ordered list of processes ("workflow steps"), so that the execution of such sequence leads to the generation of the workflow results. Workflow steps are represented in a CIF loop using _tcod_computation_* data items, and the sequential numbers of workflow steps are given in tcod_computation_step. Each step is then defined by its command line string (_tcod_computation command) and the environment variables (_tcod_computation_environment).

Input and output files and directories (artifacts in the OPM) are described in a CIF loop of _tcod_file * data items. For the sake of achieving a deterministic order, we recommend providing files and directories sorted 


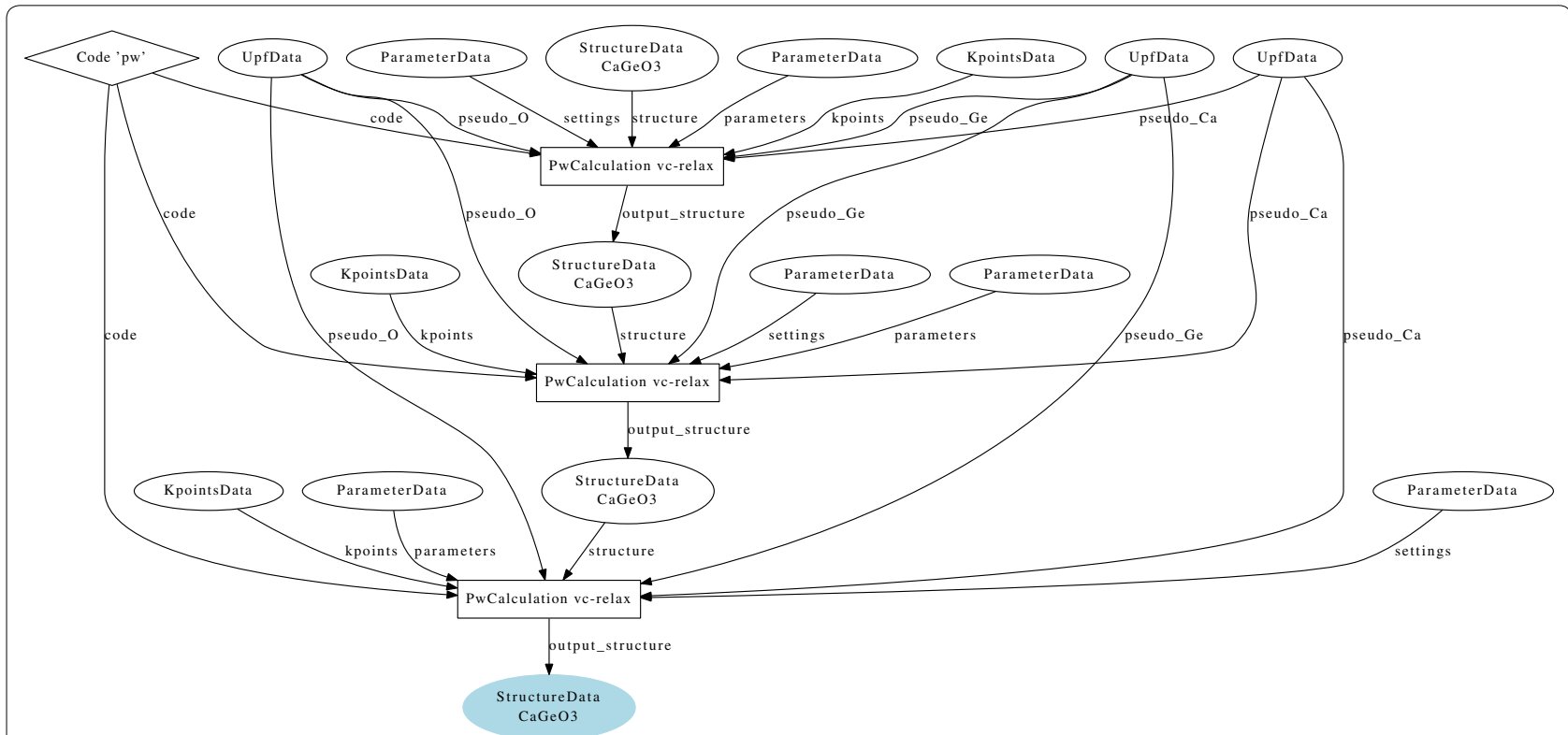

Fig. 1 Workflow of TCOD entry 10000008. The workflow consists of three consecutive relaxations of $\mathrm{GaGeO}_{3}$ structure with the Quantum ESPRESSO pw.x code. Artifacts (called "data nodes" in AiiDA) are marked as circles and processes (called "calculation nodes" in AiiDA) as rectangles. A special type of AiiDA artifact, a code, is represented by a diamond. Note that in AiiDA the direction of arrows is inverted with respect to the OPM notation $[33,34]$

by lexicographical order of their full path, with directories always preceding their contents. Moreover, all paths should be relative to the same location throughout all calculations. In particular, the data item _tcod_file name is used for names of files and directories, whereas the file content is stored in the _tcod_file_contents data item (to comply with the requirements of the CIF file format, the file contents are encoded as described in "File content inclusion in CIF and encoding" section). Since directories do not have file-like contents, the special CIF value ". (a dot, meaning "data are inapplicable") must be provided for them. Contents of standard input, output and error of processes (if any) are placed in separate files and linked to the workflow steps using tcod computation input file, tcod computation stdout and tcod_computation_stderr data items, accordingly.

While defining this format, we need to address a technical issue. The CIF format poses restrictions on acceptable CIF values, so that there are cases in which the contents of a file must be encoded (the most obvious case is a file containing a single ". symbol, that would otherwise be ignored). We have thus developed a few contentencoding protocols that we describe in detail in "File content inclusion in CIF and encoding" section, allowing inclusion of any text and binary file content into CIF text fields. An example of a simple workflow along with its representation in the TCOD CIF format is given in Fig. 2.

\section{Input data}

Initial steps of atomistic simulation workflows usually transform input data (often from external sources or databases) to internal structures. To preserve the full history, it is crucial to maintain a reference to the original data. This is relatively straightforward if the resource is available on the Internet and is assigned a permanent URI, DOI or similar resource identifier. Usually, upon retrieval, it is beneficial to supplement such identifier with retrieval date, resource version (if given) and a checksum as a tool for ultimate integrity control.

As part of this work, we have implemented a number of external database importers as part of AiiDA. These provide the user with the possibility to seamlessly import data from several structural databases, including the COD [16], ICSD [12], Material Properties Open Database (MPOD) [21], Open Quantum Materials Database (OQMD) [35], as well as a pseudopotential database, the NNIN/C Pseudopotential Virtual Vault [36]. By means of these importers, the user can both query and fetch data from the respective databases, and then import the relevant entries directly into AiiDA. During this process, we make sure to record information about the source: permanent URIs (where available), versions and checksums. When the AiiDA graph is later exported to CIF format and deposited in the TCOD, the source of the 


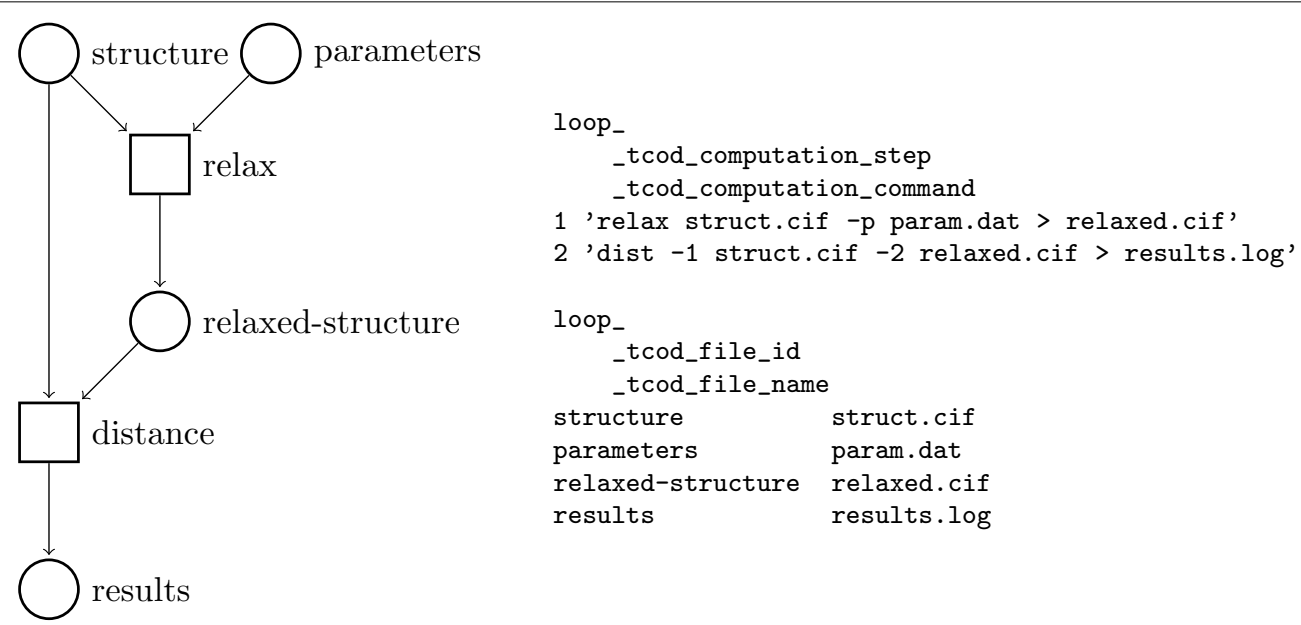

Fig. 2 Example of a simple workflow [24]. Graph view (left) and representation in TCOD CIF (right). In the graph view, files are presented as circles and processes as squares. For the sake of brevity and clarity, file contents are not reported here for the TCOD CIF representation

initial structural data is recorded in the cif_tcod. dic data items_tcod_source_structure_* and _tcod_source_database_*.

\section{File content inclusion in CIF and encoding}

As described in "Provenance model and directed acyclic graphs" section, in TCOD CIFs we aim at storing an archive of the whole computational workflow used to obtain the properties of a given material within the same TCOD CIF file. As a consequence, the file contents of individual calculation inputs and outputs are stored as values of the _tcod_file_contents data item. However, due to the format restrictions of the CIF format, not all data can be stored unmodified in a CIF 1.1 file text field. In particular, the following restrictions apply:

- the character set is restricted to printable and whitespace ASCII characters, so that CIF files must not contain unescaped binary data and Unicode symbols;

- lines (except the first) must not start with semicolons. This character is reserved as the text field delimiter. Such limitation forbids nesting CIFinside-a-CIF, but is effectively averted using line prefixing protocol [37], non-standard in CIF 1.1, albeit included in the recent CIF 2 specification [22];

- line lengths typically have to follow a number of recommendations [38].

Since no standard solution exists for the aforementioned issues of arbitrary data presentation for CIF 1.1, we have devised and implemented a protocol to encode and decode file contents prior to their storage in CIF text fields. We implemented a few encoding schemes because of their different, non-overlapping advantages:

- Numeric Character Reference (NCR): used in codtools package [38] to escape binary content and semicolons at the start of lines. This method retains the readability of the text with sparse non-ASCII symbols;

- Quoted-Printable [39]: same properties as NCR with an addition the ability to fold long lines;

- Base64 [39]: overcomes all the deficiencies of CIF at the price of readability and file size; chosen only when the file content is purely binary;

- gzip+Base64 [39, 40]: same as Base64 with additional file compression.

As one may notice, the gzip+Base64 encoding is composite: it defines a stack of two encodings-Base64encoding of gzipped contents. To accommodate this and possibly other composite encodings, we define a set of _tcod_content_encoding_* data items, allowing any complex stack of encodings to be described in a CIF loop.

The choice of the encoding is arbitrary and dependent on the requirements of readability and file size expected by the user. Nevertheless, to be able to automate the process of TCOD CIF file generation, we have implemented an algorithm to automatically choose the most appropriate encoding while trying to preserve maximum data readability, as described in "Implementation: exporting the data to the TCOD" section.

To ensure the integrity of both plain and encoded files, checksums are recorded alongside file contents. As of AiiDA v0.10.0, both MD5 and SHA1 algorithms are used. 


\section{Software versioning}

While problems of input data versioning may be avoided with revision control systems, WORM (Write Once Read Many) databases, permanent links and checksums, keeping a proper description of data transformations remains a challenging task. In particular, the algorithm of each transformation should be specified in a strictlydefined, machine-readable form. In addition, it would be extremely useful, if not essential, to be able to assert if two different representations will provide the same output when the same input is provided. Basic informatics principles, however, pose a limit on the applicability of such a description, since in general there exists no universal algorithm that could establish the equivalence of two Turing-complete language programs by formally analysing them [41, 42]. In practice, however, algorithms in normalised form could be recorded and claimed to perform identical transformations if their normalised forms are identical. This is hard to achieve, however, since these "descriptions" must be expanded to include compilation/ interpretation tools and environment, runtime operating system (OS) and external dynamically-linked libraries. Ideally these parameters should be collected recursively for every dependency. The CPU introduces a final caveat, since two different CPUs, in particular if one or both of them are buggy, can interpret the same algorithm in different ways yielding different results [43]. Thus, in addition to provenance, "descriptions" may be indispensable for an efficient bug tracking. As of AiiDA v0.10.0, data transformations are described by the internal location of executables, runtime command line parameters, environment, execution time stamp and scheduler directives. Moreover, codes from atomistic simulation packages, such as Quantum ESPRESSO [44] and NWChem [45], which are interfaced with AiiDA, can be queried for version, compilation and runtime parameters. In addition to this, manipulations in the native Python environment (referred in AiiDA as "workfunctions", or "inline calculations"), are also supplemented with the source code. In our experience, this representation is sufficient at the moment and can be easily extended in the future, for instance using virtual machines (e.g. QEMU [46], VirtualBox [47], VMware [48] or similar techniques), or Docker [49], that has rapidly become a widespread tool to reproduce a given computational environment. The presence of full process provenance information in machinereadable format would enable such reconstructions to be performed automatically.

\section{Bibliographic references}

Bibliographic references often appear in CIF files as identifiers of applied algorithms and parameters. A mechanism to provide references in a machine-readable way is described in the cif_core.dic dictionary. According to the recommendations of the International Union of Crystallography (IUCr), citation details should be given in structured tables (CIF loops). In order to categorise references according to the described aspect of the computation (force field, software code etc.), we introduce a data item tcod_citation_linkage with enumeration values of force-field, software-code, model, pseudopotential, XC-functional and basis-set, to facilitate automatic classification and filtering of computational details based on these attributes. More detailed humanreadable description of the relevance can be supplied using _citation_special_details data item.

\section{Ontologies for DFT}

The CIF dictionary cif dft.dic, developed by the advisory board of the TCOD, provides data items for the description of basis sets, pseudopotentials, atomic settings and exchange-correlation functionals. To accommodate input parameters and calculation results exported from AiiDA, we supplement the cif dft. dic dictionary with data items for Brillouin zone, kinetic energy cut-offs and calculated structure properties, such as the total energy. We also add bulk modulus and stiffness tensor, the latter being represented as a symmetric matrix of 21 independent variables. For the sake of simplicity and consistency with common practices in the core CIF dictionary, we follow the cif_core.dic's approach to represent matrices in separate "plain" data items (for example, standard anisotropic atomic displacement components in cif_core.dic's_atom site aniso U * data items) instead of relational database-style loops, used e.g. by the MPOD [21]. Figure 3 shows a sample from a TCOD CIF file, containing some newly introduced CIF data items.

The ontology defined in the cif_dft.dic dictionary is supplemented by the one that we adopt in this work. For completeness, we mention here that other projects

\begin{tabular}{|ll|}
\hline -dft_bulk_modulus & 5.95 \\
-dft_cell_energy_conv & 0.00000001 \\
-dft_BZ_integration_method & Monkhorst-Pack \\
-dft_kinetic_energy_cutoff_wavef unctions 500 \\
-dft_pseudopotential_type & PAW \\
-dft_XC_functional_type & GGA \\
-tcod_database_code & 20000419 \\
-tcod_model & DFT \\
-tcod_software_package & VASP \\
Fig.3 Sample from TCOD entry 20000419. This excerpt displays com- \\
putational setup and bulk modulus (in GPa), convergence criterion for \\
cell energy and kinetic energy cut-off for wavefunctions (both in eV). \\
Units for each data item are unambiguously defined in the TCOD \\
dictionary
\end{tabular}


have also invested effort in standardising ontologies for computational materials science simulations, especially for atomistic and/or DFT-based methods. In particular, we report a comparison of the ontology in TCOD CIF with those defined by two other projects, ETSF and NOMAD, in Tables 1 and 2, respectively. Differences are mainly in notations and conventions: for instance, ETSF uses hartree and bohr as main measurement units, NOMAD uses joule and metre, whereas the TCOD uses electronvolt and angstrom. As another example, TCOD choice of using lengths and angles of basis vectors stems from experimental crystallography, while ETSF and NOMAD use vector notation, more common in theoretical materials science. Nevertheless, automatic conversion between the two formats would be easy to implement, making it possible to seamlessly share data between different projects. The TCOD is actively working in contact with other projects to ensure the possibility of such conversion.

\section{Implementation: exporting the data to the TCOD}

The main outcome of this work is the definition and implementation of procedures to export the results of theoretical computations managed with AiiDA into CIF files and deposit them into the TCOD database. To achieve this, we have implemented a converter that, starting from a user-specified structure within the AiiDA database, is able to create a CIF format file. This converter allows for complete automatic a posteriori tagging of structures with their metadata. This is made possible by analysing the full provenance (stored in the AiiDA DAG) of the final crystal structure, extracting/ converting all relevant information, and storing it in the appropriate CIF fields defined in the TCOD dictionaries discussed before. The generation of CIF files is obtained by interfacing AiiDA with the cod-tools package [38]. We summarise here the steps of the export and deposition procedure:

1 Conversion of periodic structure from internal AiiDA representation to CIF As of AiiDA v0.10.0, there are two types of representations of periodic structures in AiiDA: structure and trajectory. A structure can be straightforwardly represented in CIF, whereas separate steps of a trajectory can be converted into structures. One or both of these conversions are used to produce an initial template CIF file (containing cif core. dic data items only), which is supplemented by additional data in the following steps.

2 Detection of the symmetry and reduction of the unit cell In AiiDA, modelled materials are represented as non-reduced unit cells of a crystal, in other words, as if their symmetry space groups were $P 1$. Such structures have to be reduced to an asymmetric unit (if possible), leaving out the symmetrically equivalent atoms. To accomplish it, we have harnessed the algorithm by Grosse-Kunstleve and Adams [50], using the implementation in spglib [51].

3 Addition of structure properties (total energies, residual forces etc.) As much data as possible is parsed from the output of a computation and added to the CIF data items defined by cif_tcod.dic and cif_dft.dic dictionaries, including energy terms and convergence criteria. We have developed a layer to convert the output parameters parsed from the computation outputs by AiiDA into data items of TCOD CIF dictionaries. Currently, the conversion is implemented for both the pw. $\mathrm{x}$ and $\mathrm{cp} . \mathrm{x}$ codes

Table 1 Comparison of a selection of TCOD CIF data items with respect to the corresponding ETSF variables

\begin{tabular}{|c|c|c|}
\hline ETSF variable & TCOD CIF data item(s) & comments \\
\hline valence_charges & _dft_atom_type_valence_electrons & \\
\hline pseudopotential_types & _dft_pseudopotential_type & \\
\hline basis_set & _dft_basisset_type & \\
\hline exchange_functional & _dft_XC_exchange_functional & \\
\hline correlation_functional & _dft_XC_correlation_functional & \\
\hline fermi_energy $\left(E_{h}\right)$ & _dft_fermi_energy $(\mathrm{eV})$ & \\
\hline smearing_scheme & $\begin{array}{l}\text { _dft_BZ_integration_smearing_method } \\
\text { _dft_BZ_integration_MP_order }\end{array}$ & $\begin{array}{l}\text { ETSF appends M-P order to the scheme, TCOD } \\
\text { CIF has a separate data item }\end{array}$ \\
\hline smearing_width & _dft_BZ_integration_smearing_width & \\
\hline kinetic_energy_cutoff $\left(E_{h}\right)$ & $\begin{array}{l}\text { _dft_kinetic_energy_cutoff_wavefunctions } \\
(\mathrm{eV})\end{array}$ & $\begin{array}{l}\text { in ETSF it is not clear whether the variable } \\
\text { applies to wavefunctions or charge densities }\end{array}$ \\
\hline kpoint_grid_shift & _dft_BZ_integration_grid_shift_[XYZ] & \\
\hline primitive_vectors $\left(a_{0}\right)$ & $\begin{array}{l}\text { _cell_length_[abc] }(\AA) \\
\text { _cell_angle_[alpha, beta,gamma] }\end{array}$ & \\
\hline $\begin{array}{l}\text { reduced_symmetry_matrices } \\
\text { reduced_symmetry_translations }\end{array}$ & _space_group_symop_operation_xyz & $\begin{array}{l}\text { ETSF provides matrices, TCOD CIF uses string } \\
\text { notation }\end{array}$ \\
\hline space_group & _space_group_IT_number & CIF has 230 spacegroups, ETSF allows for a range from 1 to 232 \\
\hline reduced_atom_positions & _atom_site_fract_[xyz] & \\
\hline atom_species & _atom_site_type_symbol & \\
\hline $\begin{array}{l}\text { atom_species_names } \\
\text { atomic_numbers } \\
\text { chemical_symbols }\end{array}$ & _atom_site_type_symbol & \\
\hline reduced_coordinates_of_kpoints & _dft_BZ_integration_grid_IBZ_point_[XYZ] & \\
\hline kpoint_weights & _dft_BZ_integration_grid_IBZ_point_weight & \\
\hline
\end{tabular}


Table 2 Comparison of a selection of TCOD CIF data items with respect to the corresponding NOMAD metadata

\begin{tabular}{|c|c|c|}
\hline NOMAD metadata & TCOD CIF data item(s) & comments \\
\hline atom_labels & _atom_site_label & \\
\hline atom_positions & _tcod_atom_site_initial_fract_[xyz] & $\begin{array}{l}\text { NOMAD uses Cartesian coordinates, TCOD uses frac- } \\
\text { tional coordinates }\end{array}$ \\
\hline basis_set_planewave_cutoff $(\mathrm{J})$ & _dft_kinetic_energy_cutoff_wavefunctions $(\mathrm{eV})$ & \\
\hline configuration_periodic_dimensions & _dft_cell_periodic_BC_[XYZ] & \\
\hline simulation_cell & $\begin{array}{l}\text { _cell_length_[abc] } \\
\text { _cell_angle_[alpha, beta,gamma] }\end{array}$ & NOMAD provides vectors \\
\hline program_compilation_datetime & _tcod_software_package_compilation_timestamp & NOMAD in Unix timestamp, TCOD CIF in ISO 8601 \\
\hline program_name & _tcod_software_package & \\
\hline program_version & _tcod_software_package_version & \\
\hline atom_forces $(\mathrm{N})$ & _tcod_atom_site_resid_force_Cartn_[xyz] $(\mathrm{eV} / \AA)$ & \\
\hline energy_total ( $\mathrm{J} /$ atom) & _tcod_total_energy (eV) & \\
\hline source_references & _tcod_source_* & $\begin{array}{l}\text { NOMAD seems to give a free-text field to identify the } \\
\text { source of data }\end{array}$ \\
\hline time_calculation & _tcod_computation_wallclock_time & \\
\hline
\end{tabular}

of Quantum ESPRESSO, as well as for the NWChem package, but the converter has been designed with a modular interface and it can thus be easily extended for any other code interfaced with AiiDA.

4 Addition of the metadata for reproduction of the results Since all metadata required for the reproduction of computations (files, scripts, command line strings, etc.) are stored by AiiDA, they are easily collected and stored within the CIF file, along with the description of each AiiDA node used in the exported workflow. Files, consisting of more than a quarter non-ASCII symbols, are assumed to be binary and encoded with Base64, whereas other files with fewer non-ASCII symbols, very long lines or other features, that could cause CIF parsing errors, are encoded with Quoted-Printable. Files larger than one kilobyte (a default value) are gzipped, if requested by the user. There is an option to exclude the contents of files that could be downloaded from the Web via provided URIs thus reducing the size of the resulting CIF file. Checksums are recorded in every case to ensure the integrity of files.

5 Deposition of generated CIF to the TCOD The final step is the upload and deposition of the CIF file in the TCOD using the HTTP protocol implemented by the TCOD. The deposition is initiated as an AiiDA calculation, wrapping the generic command line script cif_cod_deposit that is part of the cod-tools package. Cif cod deposit calls the deposition API of the TCOD and transfers the final CIF to the server for validation and deposition, if all checks are passed. The deposition step is optional, so that the final CIF can be simply exported as a local file on disk without deposition. This is useful for instance to manually inspect it before deposition, or if there is a need to share it privately.

With AiiDA installed, CIF generation can be achieved by running, on the command line, the command verdi data structure export --format tcod PK, where PK is the identifier of the structure to export. A large number of command line options exist to customise the behaviour, as explained in the AiiDA documentation. CIF deposition can be achieved instead with the command verdi data structure deposit.

\section{Discussion}

As of October 2017, the number of records in the TCOD has grown to more than 2600 . As a proof of concept, over 170 theoretical structures have been deposited to TCOD together with their provenance records using the novel AiiDA-TCOD interface presented here, constituting around $7 \%$ of current records in the TCOD. These depositions contain values of total energy in addition to more than 4600 unique AiiDA nodes, that are ready to be automatically imported into user-side AiiDA databases.

To ensure the completeness and semantic integrity of CIF files deposited to the TCOD, automatic checks are performed before accepting contributions. In fact, since CIF dictionaries contain formal descriptions of data items and their values, they can be used for automatic validation of CIF files [52]. A number of tools for automatic CIF data validation already exist, for example IUCr's checkCIF [53], iotbx's cif.validate [54] and cod-tools's cif validate. The latter is developed by the COD and TCOD development team and is the one used to validate files upon deposition. In particular, as a part of this work, additional checks have been added to cif cod check, the script (part of cod-tools) responsible for checking the semantic correctness of deposited data and for its quality control. Checks for the new data items added to the theoretical dictionaries and verification that interrelated data items (data items for coordinates; components of integration grid densities, shifts and residual forces) are simultaneously present, when expected.

Furthermore, in "File content inclusion in CIF and encoding" section we have introduced a number of encodings for files that need to be included within CIF 1.1 files. While the algorithms for decoding these 
files are known and available on the Web, it is cumbersome for a generic user to implement them in order to decode and extract the files embedded in TCOD CIFs. To address this issue, we have thus developed a program, cif_tcod_tree, to restore the full directory tree used for the execution of the simulation in AiiDA, stored in the TCOD CIF file as described in "Provenance model and directed acyclic graphs" section. After unpacking, the script further fetches remote files that are not embedded in CIF using supplied URIs. Finally, checksums are tested to ensure integrity of the files. The program is available for the end-users as part of cod-tools, and can be used to reproduce simulation results seamlessly.

To validate this feature we have asked a collaborator to reproduce the calculations of TCOD entry 10000002 using cif tcod tree. As a result of the procedure described here, the collaborator has been able to reproduce the results with great accuracy. The only differences concern the execution timings of Quantum ESPRESSO, which are inherently hardware-dependent, thus proving the robustness of our approach.

Provided that software dependencies are met, the workflow can be re-executed running each of its steps in the sequence specified in the CIF file. Finally, output results can be easily compared with the original values provided in the CIF. This makes it possible to run unsupervised replication of deposited results to automatically assess the validity of incoming data. We mention here, however, two aspects that need to be kept in mind when implementing such a service. First, running again the workflows could require a significant amount of computational time (and, for some systems or properties, it is possible to run the workflows only on large clusters). Moreover, particular care has to be taken to prevent damage, accidental or deliberate, of the system replicating the workflow, as well as illegal actions from the network. Runs should be carried out only on isolated or limited systems (i.e., software jails, virtual machines or Docker [49] images). For these reasons, fully-automated workflow replication is not yet implemented in the TCOD. However, we foresee that validation of atomistic simulations could be carried out "on the cloud" in a way very similar to continuous integration services, even harnessing existing tools and infrastructures such as Buildbot [55], Jenkins [56] or Travis-CI [57].

Finally, another important component that we have added to AiiDA, as already discussed in "Input data" section, is the set of importers from structure databases. These (and in particular those for the COD and ICSD) have been already exploited as components of workflows for materials science high-throughput investigations. As an example, in [58] the authors scanned both databases to discover, extract and screen 2D layered structures.
We expect that these tools are going to be even more useful for the computational community in the future, as they are distributed as part of the open-source software AiiDA and therefore freely available as well as extendable. As of now, users of wannier90 [59], VASP [60] and cp2k [61] simulation packages (to name a few, the full list of plug-ins is given in [62]) are able to run calculations as part of AiiDA workflows thanks to community-developed plug-ins. We anticipate that with the growing requirements for reproducibility, and with additional plug-ins for the most popular computational material science software packages, AiiDA and the TCOD will become a convenient solution for the replication problem.

\section{Conclusions}

In this article we have shown the integration of the AiiDA platform (to automatically run and manage scientific workflows while keeping full provenance of the computed data) and of the TCOD (storing computed data associated to crystal structures using an unambiguous ontology, within an open database to facilitate dissemination). Our integration makes it possible to obtain automatic a posteriori tagging of crystal structures with metadata, like computed properties and their full provenance (codes adopted, inputs used in the computation, etc.). We have first extended the TCOD CIF dictionaries for atomistic simulations, cif_tcod.dic and cif_ dft.dic, to include provenance information. We have then devised means to bypass the intrinsic limitations of the CIF 1.1 file format adopted by the TCOD. Moreover, we have implemented provenance-aware importers into AiiDA from a number of external databases for crystal structures and pseudopotentials. The main outcome of this work is the combination of all these efforts and the implementation of a converter to automatically analyse the data provenance stored in AiiDA after workflow execution, export the results into a CIF file compliant with the TCOD dictionaries, and automatically deposit it into the TCOD. Additionally, we have developed a set of tools for formal quality control and extraction of workflows from CIF files.

The general methodology described in this work does not have to be limited to the TCOD and AiiDA. It may also be implemented in other databases and frameworks, such as GNU Makefile-based replication-ready systems that have been around since as early as 1990 [63]. Our implementation proves that an automation platform to manage simulations and automatically store the full provenance of computed datasets allows metadata to be added at a later time, in a completely automated fashion. Our integration of the TCOD with AiiDA constitutes a fully-open platform implementing all four FAIR principles of "Findability, Accessibility, Interoperability, 
Reusability" for scientific data management and stewardness [64], that is furthermore fully interlinked with data generation. Indeed, our work allows the deposition in an automated fashion of computational workflows in an open database with permanent URIs and publiclyaccessible metadata/dictionaries (Findability), that can be, for instance, provided as supplementary material of computational papers. Data is available over standard protocols like HTTP (Accessibility) and, thanks to the adoption of the established CIF format and its dictionaries, both data and metadata are fully interlinked (Interoperability). Finally, data from the TCOD database together with its full provenance can be easily retrieved and imported back into AiiDA as input for further calculations and analyses (Reusability). We expect, therefore, that in the future more researchers will adopt the methods and tools described here to make the data public (as currently required by many funding agencies) with minimal required effort.

\section{Authors' contributions}

NMa and SG conceived the idea of the integration. AM developed the required software and prepared the manuscript. Developments at the AiiDA and TCOD side were supervised by GP and SG, respectively. NMo, AC and GP provided consultations concerning DFT and Quantum ESPRESSO, and contributed 170 theoretical structures for deposition to the TCOD via the novel interface. All authors contributed to the preparation of the manuscript. All authors read and approved the final manuscript.

\section{Author details}

${ }^{1}$ Theory and Simulation of Materials (THEOS) and National Centre for Computational Design and Discovery of Novel Materials (MARVEL), 1015 Lausanne, Switzerland. ${ }^{2}$ Institute of Biotechnology, Vilnius University, Sauletekio al. 7, 10257 Vilnius, Lithuania. ${ }^{3}$ Faculty of Mathematics and Informatics, Vilnius University, Naugarduko st. 24, 03225 Vilnius, Lithuania.

\section{Acknowledgements}

The authors thank Rickard Armiento for contributing theoretical data for deposition to the TCOD, Ivano E. Castelli for testing the reproduction of calculations from TCOD entry 10000002, Philippe Schwaller for developing the ICSD importer and Martin Uhrin for proofreading the manuscript.

\section{Competing interests}

The authors declare that they have no competing interests.

\section{Availability of data and materials}

The datasets supporting the conclusions of this article are available in the Crystallography Open Database, http://www.crystallography.net/cod and the Theoretical Crystallography Open Database, http://www.crystallography.net/ tcod.

AiiDA is developed in Python for Unix-like operating systems. It is released under MIT license and can be obtained free-of-charge from https://github. com/aiidateam/aiida_core. This paper refers to AiiDA v0.10.0, a snapshot of which can be obtained from https://github.com/aiidateam/aiida_core/ archive/v0.10.0.tar.gz.

cod-tools is developed in Perl and C for Unix-like operating systems. It is released under GNU GPLV2 license and can be obtained free-of-charge from svn://www.crystallography.net/cod-tools. This paper refers to cod-tools v2.1, a snapshot of which can be obtained from http://www.crystallography.net/cod/ archives/2017/software/cod-tools/cod-tools-2.1.tar.gz.

\section{Consent for publication}

Not applicable.
Ethics approval and consent to participate Not applicable.

\section{Funding}

This research was partially funded by the SCIEX grant No. 13.169, and by the MARVEL National Centre of Competence in Research of the Swiss National Science Foundation. NMa and GP acknowledge partial support from European Union H2020-EINFRA-2015-1 programme under Grant Agreement No. 676598 project "MaX - materials at the exascale".

\section{Publisher's Note}

Springer Nature remains neutral with regard to jurisdictional claims in published maps and institutional affiliations.

Received: 6 June 2017 Accepted: 1 November 2017

Published online: 14 November 2017

\section{References}

1. Jain A, Shin Y, Persson KA (2016) Computational predictions of energy materials using density functional theory. Nat Rev Mater 1(1):15004. https://doi.org/10.1038/natrevmats.2015.4

2. Jain A, Ong SP, Hautier G, Chen W, Richards WD, Dacek S, Cholia S, Gunter D, Skinner D, Ceder G, Persson KA (2013) Commentary: the materials project: a materials genome approach to accelerating materials innovation. APL Mater. 1(1):011002. https://doi.org/10.1063/1.4812323

3. Saal JE, Kirklin S, Aykol M, Meredig B, Wolverton C (2013) Materials design and discovery with high-throughput density functional theory: The Open Quantum Materials Database (OQMD). J Miner Metals Mater Soc 65:1501-1509. https://doi.org/10.1007/s11837-013-0755-4

4. Curtarolo S, Setyawan W, Wang S, Xue J, Yang K, Taylor RH, Nelson LJ, Hart GLW, Sanvito S, Buongiorno-Nardelli M, Mingo N, Levy O (2012) AFLOWLIB.ORG: a distributed materials properties repository from highthroughput ab initio calculations. Comput Mater Sci 58:227-235. https:// doi.org/10.1016/j.commatsci.2012.02.002

5. Caliste D, Pouillon Y, Verstraete MJ, Olevano V, Gonze X (2008) Sharing electronic structure and crystallographic data with ETSF_IO. Comput Phys Commun 179:748-758. https://doi.org/10.1016/j.cpc.2008.05.007

6. Gonze X, Almbladh C-O, Cucca A, Caliste D, Marques M, Freysoldt C, Olevano V, Pouillon Y, Sottile F, Verstraete M (2008) Specification of file formats for ETSF specification version 3.3. second revision for this version (SpecFF ETSF3.3). Technical report, European Theoretical Spectroscopy Facility. http://www.etsf.eu/system/files/SpecFFETSF_v3.3.pdf

7. Nomad Meta Info. https://gitlab.rzg.mpg.de/nomad-lab/ nomad-meta-info/wikis/home

8. OPTiMaDe: Open Databases Integration for Materials Design. http:// www.optimade.org

9. Theoretical Crystallography Open Database. http://www.crystallography. net/tcod/

10. Gražulis S, Merkys A, Vaitkus A, Bail AL, Chateigner D, Vilčiauskas L, Cottenier S, Björkman T, Murray-Rust P (2014) Launching the theoretical crystallography open database. Acta Cryst A 70:1736. https://doi.org/10.1107/ S2053273314082631

11. Hall SR, Allen FH, Brown ID (1991) The crystallographic information file (CIF): a new standard archive file for crystallography. Acta Crystallogr Sect A 47(6):655-685. https://doi.org/10.1107/S010876739101067X

12. Belsky A, Hellenbrandt M, Karen VL, Luksch P (2002) New developments in the Inorganic Crystal Structure Database (ICSD): accessibility in support of materials research and design. Acta Crystallogr Sect B 58(3 Part 1):364-369. https://doi.org/10.1107/S0108768102006948

13. Groom CR, Allen FH (2014) The Cambridge Structural Database in retrospect and prospect. Angew Chem Int Ed 53:662-671. https://doi. org/10.1002/anie.201306438

14. Rajan H, Uchida H, Bryan DL, Swaminathan R, Downs RT, Hall-Wallace M (2006) Building the american mineralogist crystal structure database: a recipe for construction of a small internet database. In: Sinha AK (ed) Geoinformatics: data to knowledge. Geological Society of America 
Special Papers, vol 397, pp 73-80. Geological Society of America, Boulder, CO, United States. https://doi.org/10.1130/2006.2397(06)

15. Crystallography Open Database. http://www.crystallography.net/cod/

16. Gražulis S, Daškevič A, Merkys A, Chateigner D, Lutterotti L, Quirós M, Serebryanaya NR, Moeck P, Downs RT, Le Bail A (2012) Crystallography Open Database (COD): an open-access collection of crystal structures and platform for world-wide collaboration. Nucleic Acids Res 40(D1):420-427. https://doi.org/10.1093/nar/gkr900

17. Brown ID, McMahon B (2002) CIF: the computer language of crystallography. Acta Crystallogr Sect B 58:317-324. https://doi.org/10.1107/ S0108768102003464

18. Fitzgerald PMD, Westbrook JD, Bourne PE, McMahon B, Watenpaugh KD, Berman HM (2006) Chap. 4.5. Macromolecular dictionary (mmCIF). In: Hall SR, McMahon B (eds) International tables for crystallography, vol G, pp. 295-443. International Union of Crystallography. https://doi.org/10.1107/ 97809553602060000745

19. Toby BH, Von Dreele RB, Larson AC (2003) CIF applications. XIV. reporting of Rietveld results using pdCIF: GSAS2CIF. J Appl Crystallogr 36:1290-1294

20. Mallinson PR, Brown ID (2005) In: Hall SR, McMahon B (eds) International tables for crystallography, vol G, pp 141-143. International Union of Crystallography

21. Pepponi G, Gražulis S, Chateigner D (2012) MPOD: A Material Property Open Database linked to structural information. Nucl Instrum Methods Phys Res Sect B Beam Interact Mater Atoms 284(0):10-14. https://doi. org/10.1016/..nimb.2011.08.070. E-MRS 2011 Spring Meeting, Symposium $\mathrm{M}: \mathrm{X}$-ray techniques for materials research-from laboratory sources to free electron lasers

22. Bernstein HJ, Bollinger JC, Brown ID, Gražulis S, Hester JR, McMahon B, Spadaccini N, Westbrook JD, Westrip SP (2016) Specification of the Crystallographic Information File format, version 2.0. J Appl Crystallogr 49(1):277-284. https://doi.org/10.1107/s1600576715021871

23. AiiDA: Automated Interactive Infrastructure and Database for Computational Science. http://www.aiida.net

24. Pizzi G, Cepellotti A, Sabatini R, Marzari N, Kozinsky B (2016) AiiDA: automated interactive infrastructure and database for computational science. Comput Mater Sci 111:218-230. https://doi.org/10.1016/j. commatsci.2015.09.013

25. TCOD Mailing List. http://lists.crystallography.net/cgi-bin/mailman/ listinfo/tcod

26. Mesirov JP (2010) Computer science. Accessible reproducible research. Science (New York, N.Y.) 327(5964):415-416. https://doi.org/10.1126/ science. 1179653

27. Peng RD, Dominici F, Zeger SL (2006) Reproducible epidemiologic research. Am J Epidemiol 163(9):783-789. https://doi.org/10.1093/aje/ kwj093. http://aje.oxfordjournals.org/content/163/9/783.full.pdf+html

28. Peng RD (2009) Reproducible research and biostatistics. Biostatistics 10(3):405-408. https://doi.org/10.1093/biostatistics/kxp014. http://biostatistics.oxfordjournals.org/content/10/3/405.full.pdf +html

29. Peng RD (2011) Reproducible research in computational science. Science 334(6060):1226-1227. https://doi.org/10.1126/science.1213847. http:// www.sciencemag.org/content/334/6060/1226.full.pdf

30. Peng RD, Eckel SP (2008) Distributed reproducible research using cached computations. http://www.biostat.jhsph.edu/\$ \$rpeng/papers/archive/ distRR.pdf. Accessed 06 June 2015

31. Leisch F (2008) Sweave user manual. http://www.stat.uni-muenchen. de/\$ \$leisch/Sweave/Sweave-manual.pdf

32. Team R Core (2017) R: A language and environment for statistical computing. R Foundation for Statistical Computing, Vienna, Austria. https:// www.R-project.org

33. Moreau L, Freire J, Futrelle J, McGrath RE, Myers J, Paulson P (2007) The open provenance model. Technical report, University of Southampton. http://eprints.soton.ac.uk/264979/

34. Moreau L, Freire J, Futrelle J, McGrath RE, Myers J, Paulson P (2008) The open provenance model: an overview. In: Freire J, Koop D, Moreau L (eds) Provenance and annotation of data and processes. Lecture Notes in Computer Science, vol 5272. Springer, Berlin, pp 323-326. https://doi. org/10.1007/978-3-540-89965-5_31

35. Kirklin S, Saal JE, Meredig B, Thompson A, Doak JW, Aykol M, Rühl S, Wolverton C (2015) The Open Quantum Materials Database (OQMD):
Assessing the accuracy of DFT formation energies. npj Comput Mater 1(1):15010. https://doi.org/10.1038/npjcompumats.2015.10

36. NNIN/C Pseudopotential Virtual Vault. http://nninc.cnf.cornell.edu/

37. Bollinger J. CIF-changes to the specification. http://www.iucr.org/_data/ assets/pdf_file/0020/59420/cif2_syntax_changes-jcb20110728.pdf

38. Merkys A, Vaitkus A, Butkus J, Okulič-Kazarinas M, Kairys V, Gražulis S (2016) COD::CIF::Parser: an error-correcting CIF parser for the Perl language. J Appl Crystallogr 49(1):292-301. https://doi.org/10.1107/ S1600576715022396

39. Freed N, Borenstein N (1996) Multipurpose internet mail extensions (MIME) part one: format of internet message bodies. Technical report. https://tools.ietf.org/html/rfc2045

40. Deutsch P (1996) GZIP file format specification version 4.3. Technical report. https://tools.ietf.org/html/rfc1952

41. Turing AM (1937) On computable numbers, with an application to the Entscheidungsproblem. Proc Lond Math Soc s2-42(1):230-265. https:// doi.org/10.1112/plms/s2-42.1.230

42. Rice HG (1953) Classes of recursively enumerable sets and their decision problems. Trans Am Math Soc 74(2):358-358. https://doi.org/10.1090/ s0002-9947-1953-0053041-6

43. Moler C (1995) A tale of two numbers. MATLAB News and Notes, pp $10-12$

44. Giannozzi P, Baroni S, Bonini N, Calandra M, Car R, Cavazzoni C, Ceresoli D, Chiarotti GL, Cococcioni M, Dabo I, Corso AD, de Gironcoli S, Fabris S, Fratesi G, Gebauer R, Gerstmann U, Gougoussis C, Kokalj A, Lazzeri M, Martin-Samos L, Marzari N, Mauri F, Mazzarello R, Paolini S, Pasquarello A, Paulatto L, Sbraccia C, Scandolo S, Sclauzero G, Seitsonen AP, Smogunov A, Umari P, Wentzcovitch RM (2009) QUANTUM ESPRESSO: a modular and open-source software project for quantum simulations of materials. J Phys Condens Matter 21(39):395502. https://doi. org/10.1088/0953-8984/21/39/395502

45. Valiev M, Bylaska EJ, Govind N, Kowalski K, Straatsma TP, Dam HJJV, Wang D, Nieplocha J, Apra E, Windus TL, de Jong WA (2010) NWChem: a comprehensive and scalable open-source solution for large scale molecular simulations. Comput Phys Commun 181(9):1477-1489. https://doi. org/10.1016/j.cpc.2010.04.018

46. QEMU. http://www.qemu-project.org

47. VirtualBox. https://www.virtualbox.org

48. VMware ESXi. http://www.vmware.com/products/esxi-and-esx.html

49. Docker. https://www.docker.com

50. Grosse-Kunstleve RW, Adams PD (2002) Algorithms for deriving crystallographic space-group information. II. Treatment of special positions. Acta Crystallogr A 58(Pt 1):60-65

51. Spglib. https://atztogo.github.io/spglib/

52. McMahon B (2006) Chap. 3.1. General considerations when defining a CIF data item. In: Hall, S.R., McMahon, B. (eds.) International Tables for Crystallography, vol. G, pp. 73-91. International Union of Crystallography, (IUCr). https://doi.org/10.1107/97809553602060000733. http://xrpp.iucr.org/Ga/ ch3o1v0001/

53. checkCIF. http://checkcif.iucr.org

54. Gildea RJ, Bourhis LJ, Dolomanov OV, Grosse-Kunstleve RW, Puschmann H, Adams PD, Howard JAK (2011) iotbx.cif: a comprehensive CIF toolbox. J Appl Crystallogr 44(6):1259-1263. https://doi.org/10.1107/ S0021889811041161

55. Buildbot. http://buildbot.net

56. Jenkins: An Extensible Open Source Continuous Integration Server. http://jenkins-ci.org

57. Travis Cl. https://travis-ci.org

58. Mounet N, Gibertini M, Schwaller P, Campi D, Merkys A, Marrazzo A, Sohier T, Castelli IE, Cepellotti A, Pizzi G, Marzari N (2016) Novel twodimensional materials from high-throughput computational exfoliation of experimentally known compounds. arXiv: org/abs/1611.05234v1

59. Mostofi AA, Yates JR, Pizzi G, Lee Y-S, Souza I, Vanderbilt D, Marzari N (2014) An updated version of wannier90: a tool for obtaining maximallylocalised Wannier functions. Comput Phys Commun 185(8):2309-2310. https://doi.org/10.1016/..cpc.2014.05.003

60. Kresse G, Furthmüller J (1996) Efficient iterative schemes forab initiototal-energy calculations using a plane-wave basis set. Phys Rev B 54(16):11169-11186. https://doi.org/10.1103/physrevb.54.11169 
61. Hutter J, lannuzzi M, Schiffmann F, VandeVondele J (2013) cp2k: atomistic simulations of condensed matter systems. Wiley Interdiscip Rev Comput Mol Sci 4(1):15-25. https://doi.org/10.1002/wcms.1159

62. Registry for Aiida Plugins. https://github.com/aiidateam/aiida-registry

63. Schwab M, Karrenbach N, Claerbout J (2000) Making scientific computations reproducible. Comput Sci Eng 2:61-67. https://doi. org/10.1109/5992.881708

64. Wilkinson MD, Dumontier M, Aalbersberg IJ, Appleton G, Axton M, Baak A, Blomberg N, Boiten J-W, da Silva Santos LB, Bourne PE, Bouwman J, Brookes AJ, Clark T, Crosas M, Dillo I, Dumon O, Edmunds S, Evelo CT, Finkers R, Gonzalez-Beltran A, Gray AJG, Groth P, Goble C, Grethe JS, Heringa J, ât Hoen PAC, Hooft R, Kuhn T, Kok R, Kok J, Lusher SJ, Martone ME, Mons
A, Packer, AL, Persson B, Rocca-Serra P, Roos M, van Schaik R, Sansone S.-A, Schultes E, Sengstag T, Slater T, Strawn G, Swertz MA, Thompson $M$, van der Lei J, van Mulligen E, Velterop J, Waagmeester A, Wittenburg P, Wolstencroft K, Zhao J, Mons B (2016) The fair guiding principles for scientific data management and stewardship. Scientific Data 3, 160018

\section{Submit your manuscript to a SpringerOpen ${ }^{\circ}$ journal and benefit from:}

- Convenient online submission

- Rigorous peer review

- Open access: articles freely available online

- High visibility within the field

- Retaining the copyright to your article

Submit your next manuscript at $\mathbf{s p r i n g e r o p e n . c o m ~}$ 